\title{
SEMICOMPATIBILITY AND FIXED POINT THEOREMS IN AN UNBOUNDED D-METRIC SPACE
}

\author{
BIJENDRA SINGH, SHISHIR JAIN, AND SHOBHA JAIN
}

Received 16 March 2004 and in revised form 24 August 2004

Rhoades (1996) proved a fixed point theorem in a bounded $D$-metric space for a contractive self-map with applications. Here we establish a more general fixed point theorem in an unbounded $D$-metric space, for two self-maps satisfying a general contractive condition with a restricted domain of $x$ and $y$. This has been done by using the notion of semicompatible maps in $D$-metric space. These results generalize and improve the results of Rhoades (1996), Dhage et al. (2000), and Veerapandi and Rao (1996). These results also underline the necessity and importance of semicompatibility in fixed point theory of $D$-metric spaces. All the results of this paper are new.

\section{Introduction}

There have been a number of generalizations of metric spaces. One such generalization is generalized metric space or D-metric space initiated by Dhage [1] in 1992. He proved some results on fixed points for a self-map satisfying a contraction for complete and bounded $D$-metric spaces. Rhoades [4] generalized Dhage's contractive condition by increasing the number of factors and proved the existence of unique fixed point of a selfmap in $D$-metric space. Recently, motivated by the concept of compatibility for metric space, Singh and Sharma [5] introduced the concept of $D$-compatibility of maps in $D$ metric space and proved some fixed point theorems using a contractive condition.

In [4], the following theorem has been established.

Theorem 1.1 (Rhoades [4]). Let $X$ be a complete and bounded D-metric space, and let $T$ be a self-map of $X$ satisfying $D(T x, T y, T z) \leq k \operatorname{Max}\{D(x, y, z), D(x, T x, z), D(y, T y, z)$, $D(x, T y, z), D(y, T x, z)\}$, for all $x, y, z$ in $X, 0 \leq k<1$. Then $T$ has a unique fixed point $p$ in $X$ and $T$ is continuous at $p$.

The object of this paper is to generalize this contraction by increasing the number of factors in it from five to ten. We involve two semicompatible self-maps in it, one of which is continuous. Further, the domain of $x$ and $y$ from the whole space has been reduced to some orbits only. The first result gives a sufficient condition for existence of a unique fixed point with $T$ is continuous and $(S, T)$ semicompatible and the second one gives 
the same result if $S$ continuous. For the sake of completeness, following Dhage $[1,2]$, we recall some definitions and known results in $D$-metric space.

\section{Preliminaries}

In what follows $(X, D)$ will denote a $D$-metric space, $\mathbb{N}$ the set of all natural numbers, and $\mathbb{R}^{+}$the set of all positive real numbers.

Definition 2.1. Let $X$ be a nonempty set. A generalized metric (or $D$-metric) on $X$ is a function: $D: X \times X \times X \rightarrow \mathbb{R}^{+}$that satisfies the following conditions.

$(D-1) D(x, y, z)=0$ iff $x=y=z$ (sufficiency).

$(D-2) D(x, y, z)=D(p\{x, y, z\})$, (symmetry) where $p$ is a permutation function.

$(D-3) D(x, y, z) \leq D(x, y, a)+D(x, a, z)+D(a, y, z)$, for all $x, y, z$, and $a$ in $X$ (tetrahedral inequality).

The pair $(X, D)$ is called a generalized metric (or $D$-metric) space. Geometrically a $D$ metric $D(x, y, z)$ represents the perimeter of a triangle whose vertices are $x, y$, and $z$. Immediate examples of such a function are

(a) $D(x, y, z)=\operatorname{Max}\{d(x, y), d(y, z), d(x, z)\}$,

(b) $D(x, y, z)=d(x, y)+d(y, z)+d(x, z)$.

Here, $d$ is the ordinary metric on $X$.

Definition $2.2[1]$. A sequence $\left\{x_{n}\right\}$ in a $D$-metric space is said to $D$-converge to a point $x$ if for any $\varepsilon>0$, there exists a positive integer $n_{0}$ such that $D\left(x_{n}, x_{m}, x\right)<\varepsilon$, for all $n, m>n_{0}$. A sequence $\left\{x_{n}\right\}$ is said to be a $D$-Cauchy sequence if for each $\varepsilon>0$ there exists $n_{0} \in \mathbb{N}$ such that $D\left(x_{n}, x_{n+p}, x_{n+p+t}\right)<\varepsilon$, for all $n>n_{0}$, for all $p, t \in \mathbb{N}$. $X$ is said to be complete if each Cauchy sequence of it converges to some point of $X$.

In a $D$-metric space, if $D$ is continous in two variables, then the limit of sequence is unique, if it exists. Throughout this paper the $D$-metric is assumed to be continous in two variables.

Definition 2.3. Let $(X, D)$ be a $D$-metric space and $S$ be a nonempty subset of $X$. We define the diameter of $S$ as $\delta_{d}(S)=\operatorname{Sup}\{D(x, y, z) \mid x, y, z \in S\}$.

Definition 2.4 [6]. Let $T$ be a multi-valued map on $D$-metric space $(X, D)$. Let $x_{0} \in X$. A sequence $\left\{x_{n}\right\}$ in $X$ is said to be an orbit of $T$ at $x_{0}$ denoted by $O\left(T, x_{0}\right)$ if $x_{n-1} \in T^{n-1}\left(x_{0}\right)$, that is, $x_{n} \in T x_{n-1}$, for all $n \in \mathbb{N}$. The set of all orbits of $T$ at $x_{0}$ is denoted by $F\left(T, x_{0}\right)$. An orbit $O\left(T, x_{0}\right)$ is said to be bounded if its diameter is finite.

Definition 2.5 [5]. Self maps $S$ and $T$ on a $D$-metric space $(X, D)$ are said to be $D$ compatible if $\lim _{n \rightarrow \infty} D\left(S T x_{n}, T S x_{n}, z\right)=0$, where $z=S T x_{n}$ or $T S x_{n}$, when ever $\left\{x_{n}\right\}$ is a sequence in $X$ such that $\lim _{n \rightarrow \infty} T x_{n}=\lim _{n \rightarrow \infty} S x_{n}=x \in X$.

Definition 2.6. A pair $(S, T)$ of self-mappings of a $D$-metric space is said to be semicompatible if $\lim _{n \rightarrow \infty} S T x_{n}=T x$, when ever $\left\{x_{n}\right\}$ is a sequence in $X$ such that $\lim _{n \rightarrow \infty} T x_{n}$ $=\lim _{n \rightarrow \infty} S x_{n}=x \in X$.

It follows that $(S, T)$ is semicompatible and $S y=T y$ then $S T y=T S y$. 
Propostion 2.7. S and T are D-compatible self-maps on a D-metric space $(X, D)$ and $T$ is continuous then the pair $(S, T)$ is semicompatible.

Proof. Let $\left\{S x_{n}\right\} \rightarrow u,\left\{T x_{n}\right\} \rightarrow u$ for some $u \in X$. To show this, $S T x_{n} \rightarrow T u$. As $T$ is continuous $T S x_{n} \rightarrow T u$.

Now, as $(S, T)$ is $D$-compatible we have $\lim _{n \rightarrow \infty} D\left(S T x_{n}, S T x_{n}, T S x_{n}\right)=0$. That is, $\lim _{n \rightarrow \infty} D\left(S T x_{n}, S T x_{n+p}, T S x_{n}\right)=0$. That is, $\lim _{n \rightarrow \infty} D\left(S T x_{n}, S T x_{n+p}, T u\right)=0$. That is, $\lim _{n \rightarrow \infty} S T x_{n}=T u$. Hence $(S, T)$ is semicompatible.

Propostion 2.8. S and T are semicompatible self-maps on a D-metric space $(X, D)$ and $T$ is continuous then $(S, T)$ is D-compatible.

Proof. Let $\left\{S x_{n}\right\} \rightarrow u,\left\{T x_{n}\right\} \rightarrow u$ and as $T$ is continuous $T S x_{n} \rightarrow T u$. The semicompatibility of $(S, T)$ gives $S T x_{n} \rightarrow T u$. Now, $\lim _{n \rightarrow \infty} D\left(S T x_{n}, S T x_{n}, T S x_{n}\right)=D(T u, T u$, $T u)=0$. Hence $(S, T)$ is $D$-compatible.

The following is an example of a pair of self-maps $(S, T)$ which is semicompatible but not compatible. Further, it is shown that the semicompatibility of the pair $(S, T)$ need not imply the semicompatibility of $(T, S)$.

Example 2.9. Let $X=[0,1]$ and consider the $D$-metric space $(X, D)$, where $D$ is defined by $D(x, y, z)=\operatorname{Max}\{|x-y|,|y-z|,|z-x|\}$, for all $x, y, z \in X$. Define a self-map as follows:

$$
\begin{gathered}
S x=x \quad \text { if } 0 \leq x<\frac{1}{2}, \\
S x=1 \quad \text { if } x \geq \frac{1}{2} .
\end{gathered}
$$

Let $I$ be the identity map on $X$ and $x_{n}=1 / 2-1 / n$. Then $\left\{I x_{n}\right\}=\left\{x_{n}\right\} \rightarrow 1 / 2$ and $\left\{S x_{n}\right\} \rightarrow$ $1 / 2$. Again, $\left\{I S x_{n}\right\}=\left\{S x_{n}\right\} \rightarrow 1 / 2 \neq S(1 / 2)$. Thus $(I, S)$ is not semicompatible though it is compatible. Also for any sequence $\left\{x_{n}\right\}$ in $X$ such that $\left\{x_{n}\right\} \rightarrow x$ and $\left\{S x_{n}\right\} \rightarrow x$ we have $\left\{S I x_{n}\right\}=\left\{S x_{n}\right\} \rightarrow x=I x$. Thus $(S, I)$ is always semicompatible.

Remark 2.10. The above example gives an important aspect of semicompatibility in $D$ metric space as the pair $(I, S)$ is commuting, weakly commuting, compatible, and weak compatible, still it is not semicompatible.

The following is an example of a pair of maps which is semicompatible but not compatible.

Example 2.11. Let $X=[0,2]$, define $D(x, y, z)=\operatorname{Max}\{|x-y|,|y-z|,|z-x|\}$, for all $x, y, z \in X$. Define self-maps $A$ and $S$ on $X$ as follows:

$$
\begin{aligned}
S x=1, & & x \in[0,1), \\
2, & & x=1, \\
\frac{x+3}{5}, & & x \in(1,2], \\
A x=2, & & x \in[0,1], \\
\frac{x}{2}, & & x \in(1,2] .
\end{aligned}
$$


Take $x_{n}=2-1 /(2 n)$ then we have $S(1)=A(1)=2$ and $S(2)=A(2)=1$. Also $S A(1)=$ $A S(1)=1$ and $S A(2)=A S(2)=2$. Hence $A x_{n} \rightarrow 1$ and $S x_{n} \rightarrow 1, A S x_{n} \rightarrow 2$, and $S A x_{n} \rightarrow 1$. Now,

$$
\begin{gathered}
\lim _{n \rightarrow \infty} D\left(A S x_{n}, A S x_{m}, S y\right)=D(2,2,2)=0, \\
\lim _{n \rightarrow \infty} M\left(A S x_{n}, S A x_{n}, A S x_{n}\right)=D(2,1,2)=1 \neq 0 .
\end{gathered}
$$

Hence $(A, S)$ is semicompatible but it is not compatible.

Propostion 2.12. Let $S$ and $T$ be two self-maps of a D-metric space $(X, D)$ such that $S(X) \subseteq$ $T(X)$. For some $x_{0} \in X$ define sequences $\left\{x_{n}\right\}$ and $\left\{y_{n}\right\}$ in $X$ by $S x_{n-1}=T x_{n}=y_{n}$, for all $n \in \mathbb{N}$. Then

(i) $O\left(T^{-1} S, x_{0}\right)=\left\{x_{0}, x_{1}, x_{2}, x_{3}, \ldots, x_{n}, \ldots\right\}$,

(ii) $O\left(S T^{-1}, S x_{0}\right)=\left\{y_{1}, y_{2}, y_{3}, \ldots, y_{n}, \ldots\right\}$.

Proof. As $S x_{0}=T x_{1}$ we have $x_{1} \in T^{-1} S x_{0}$ and $S x_{1}=T x_{2}$ gives $x_{2} \in T^{-1} S x_{1}=\left(T^{-1} S\right)^{2} x_{0}$. Similarly, $S x_{n-1}=T x_{n}$ gives $x_{n} \in T^{-1} S x_{n-1}=\left(T^{-1} S\right)^{n} x_{0}$. Again,

$y_{1}=S x_{0}, \quad y_{2}=S x_{1} \in S T^{-1} S x_{0}=\left(S T^{-1}\right) S x_{0}, \quad y_{3}=S x_{2} \in S T^{-1} S T^{-1} S x_{0}=\left(S T^{-1}\right)^{2} S x_{0}$.

Similarly, $y_{n} \in\left(S T^{-1}\right)^{n-1} S x_{0}$.

Let $\varphi$ denote the class of functions $\phi: \mathbb{R}^{+} \rightarrow \mathbb{R}^{+}$which are upper semicontinuous nondecreasing and $\phi(t)<t$, for $t>0$. If the orbit $\left\{y_{n}\right\}$ is bounded, define $\gamma_{i}=\delta_{d}\left\{y_{i}, y_{i+1}\right.$, $\left.y_{i+2}, \ldots\right\}, i=1,2, \ldots$. Then $\gamma_{n}$ is finite for all $n$ and also $\left\{\gamma_{n}\right\}$ is a nonincreasing sequence and $\gamma_{n} \geq 0$, for all $n$. Hence $\gamma_{n} \rightarrow \gamma(\gamma \geq 0)$ as $n \rightarrow \infty$.

LeMmA 2.13. Let $S, T, x_{n}, y_{n}, x_{0}$ be as above. If

(i) some orbit $\left\{y_{n}\right\}=O\left(S T^{-1}, S x_{0}\right)$ is bounded,

(ii) for all $x, y, z$ in $O\left(T^{-1} S, x_{0}\right)$, for some $\phi \in \varphi$,

$$
\begin{aligned}
D(S x, S y, S z) \leq \phi \operatorname{Max}\{ & D(T x, T y, T z), D(S x, T x, T z), D(S y, T y, T z), D(S x, T y, T z), \\
& D(S y, T x, T z), D(T x, T y, S z), D(S x, T x, S z), \\
& D(S y, T y, S z), D(S x, T y, S z), D(S y, T x, S z)\}
\end{aligned}
$$

then $\lim _{n \rightarrow \infty} \gamma_{n}=0$ and $\left\{y_{n}\right\}$ is a D-Cauchy sequence in $O\left(S T^{-1}, S x_{0}\right)$. 
Proof. We have from condition (ii)

$$
\begin{aligned}
& D\left(y_{n}, y_{n+p}, y_{n+p+t}\right)=D\left(S x_{n-1}, S x_{n+p-1}, S x_{n+p+t-1}\right) \\
& \leq \phi \operatorname{Max}\left\{D\left(y_{n-1}, y_{n+p-1}, y_{n+p+t-1}\right), D\left(y_{n-1}, y_{n}, y_{n+p+t-1}\right),\right. \\
& D\left(y_{n+p-1}, y_{n+p}, y_{n+p+t-1}\right), D\left(y_{n}, y_{n+p-1}, y_{n+p+t-1}\right), \\
& D\left(y_{n-1}, y_{n+p}, y_{n+p+t-1}\right), D\left(y_{n-1}, y_{n+p-1}, y_{n+p+t}\right) \text {, } \\
& D\left(y_{n-1}, y_{n}, y_{n+p+t}\right), D\left(y_{n+p-1}, y_{n+p}, y_{n+p+t}\right) \text {, } \\
& \left.D\left(y_{n}, y_{n+p-1}, y_{n+p+t}\right), D\left(y_{n-1}, y_{n+p}, y_{n+p+t}\right)\right\} \\
& \leq \phi\left(\gamma_{n-1}\right) \text {. }
\end{aligned}
$$

Taking sup over $p$ and $t$ we have $\gamma_{n} \leq \phi\left(\gamma_{n-1}\right)$ and letting $n \rightarrow \infty$ we get $\gamma \leq \phi(\gamma)<\gamma$ if $\gamma>0$, which is a contradiction. Hence $\gamma=0$, that is, $\gamma_{n} \rightarrow 0$, as $n \rightarrow \infty$.

Thus, $D\left(y_{n}, y_{n+p}, y_{n+p+t}\right) \leq \phi\left(\gamma_{n-1}\right) \rightarrow 0$ as $n \rightarrow \infty$. Hence $\left\{y_{n}\right\}$ is a $D$-Cauchy sequence.

\section{Main results}

Theorem 3.1. Let $S$ and $T$ be self-maps of a D-metric space $(X, D)$ satisfying the following.

(1) $S(X) \subseteq T(X)$.

(2) The pair $(S, T)$ is semicompatible and $T$ is continuous.

(3) For some $x_{0} \in X$, some orbit $\left\{y_{n}\right\}=O\left(S T^{-1}, S x_{0}\right)$ is bounded and complete.

(4) For all $x, y \in O\left(T^{-1} S, x_{0}\right) \cup O\left(S T^{-1}, S x_{0}\right)$ and for all $z \in X$ and for some $\phi \in \varphi$,

$$
\begin{aligned}
D(S x, S y, S z) \leq \phi \operatorname{Max}\{ & D(T x, T y, T z), D(S x, T x, T z), D(S y, T y, T z), \\
& D(S x, T y, T z), D(S y, T x, T z), D(T x, T y, S z), \\
& D(S x, T x, S z), D(S y, T y, S z), D(S x, T y, S z), \\
& D(S y, T x, S z)\} .
\end{aligned}
$$

Then $S$ and $T$ have a unique common fixed point.

Proof. For $x_{0} \in X$, construct sequences $\left\{x_{n}\right\}$ and $\left\{y_{n}\right\}$ in $X$ as $S x_{n-1}=T x_{n}=y_{n}$, for all $n \in \mathbb{N}$. Then by Lemma 2.13, $\left\{y_{n}\right\}$ is a Cauchy sequence in $O\left(S T^{-1}, S x_{0}\right)$ which is complete. Hence,

$$
y_{n}=T x_{n}=S x_{n-1} \longrightarrow u \in X
$$

As $T$ is continuous and $(S, T)$ is semicompatible we get

$$
T^{2} x_{n} \longrightarrow T u, \quad S T x_{n} \longrightarrow T u .
$$


794 Semicompatibility in unbounded $D$-metric space

Step 1. Putting $x=T x_{n}, y=T x_{n}, z=x_{n}$ in condition (4) we get

$$
\begin{aligned}
D\left(S T x_{n}, S T x_{n}, S x_{n}\right) \leq \phi M \operatorname{Max} & D\left(T T x_{n}, T T x_{n}, T x_{n}\right), D\left(S T x_{n}, T T x_{n}, T x_{n}\right), \\
& D\left(S T x_{n}, T T x_{n}, T x_{n}\right), D\left(S T x_{n}, T T x_{n}, T x_{n}\right), \\
& D\left(S T x_{n}, T T x_{n}, T x_{n}\right), D\left(T T x_{n}, T T x_{n}, S x_{n}\right), \\
& D\left(S T x_{n}, T T x_{n}, S x_{n}\right), D\left(S T x_{n}, T T x_{n}, S x_{n}\right), \\
& \left.D\left(S T x_{n}, T T x_{n}, S x_{n}\right), D\left(S T x_{n}, T T x_{n}, S x_{n}\right)\right\} .
\end{aligned}
$$

Taking limit as $n \rightarrow \infty$ and using (3.2) and (3.3) we get

$$
D(T u, T u, u) \leq \phi\{D(T u, T u, u)\} \Longrightarrow T u=u .
$$

Step 2. Putting $x=x_{n}, y=x_{n}$, and $z=u$ in condition (4) we get

$$
\begin{aligned}
D\left(S x_{n}, S x_{n}, S u\right) \leq \phi M \operatorname{Max}\{ & D\left(T x_{n}, T x_{n}, T u\right), D\left(S x_{n}, T x_{n}, T u\right), D\left(S x_{n}, T x_{n}, T u\right), \\
& D\left(S x_{n}, T x_{n}, T u\right), D\left(S x_{n}, T x_{n}, T u\right), D\left(T x_{n}, T x_{n}, S u\right), \\
& D\left(S x_{n}, T x_{n}, S u\right), D\left(S x_{n}, T x_{n}, S u\right), D\left(S x_{n}, T x_{n}, S u\right), \\
& \left.D\left(S x_{n}, T x_{n}, S u\right)\right\} .
\end{aligned}
$$

Taking limit as $n \rightarrow \infty$ using (3.2) and (3.5) we get

$$
D(u, u, S u) \leq \phi\{D(u, u, S u)\}<D(u, u, S u) \quad \text { if } D(u, u, S u)>0,
$$

which is a contradiction, hence $D(u, u, S u)=0$, that is, $u=S u$. Hence $u=S u=T u$. That is, $u$ is a common fixed point of $S$ and $T$.

Step 3 (Uniqueness). Let $w$ be another common fixed point of $S$ and $T$, then

$$
w=S w=T w
$$

Putting $x=x_{n}, y=x_{n}$, and $z=w$ in condition (4) we get

$$
\begin{aligned}
D\left(S x_{n}, S x_{n}, S w\right) \leq \phi M \operatorname{Max}\{ & D\left(T x_{n}, T x_{n}, T w\right), D\left(S x_{n}, T x_{n}, T w\right), D\left(S x_{n}, T x_{n}, T w\right), \\
& D\left(S x_{n}, T x_{n}, T w\right), D\left(S x_{n}, T x_{n}, T w\right), D\left(T x_{n}, T x_{n}, S w\right), \\
& D\left(S x_{n}, T x_{n}, S w\right), D\left(S x_{n}, T x_{n}, S w\right), D\left(S x_{n}, T x_{n}, S w\right), \\
& \left.D\left(S x_{n}, T x_{n}, S w\right)\right\} .
\end{aligned}
$$

Taking limit as $n \rightarrow \infty$ using (3.2) to (3.8) we get $D(u, u, w) \leq \phi\{D(u, u, w)\}<D(u, u, w)$ if $D(u, u, w)>0$ which is a contradiction, hence $D(u, u, w)=0$, that is, $u=w$. Hence, $u$ is a unique common fixed point of $S$ and $T$.

Remark 3.2. It is clear from the above proof that the satisfaction of condition (4) of Theorem 3.1 for all $x, y \in O\left(T^{-1} S, x_{0}\right) \cup O\left(S T^{-1}, S x_{0}\right)$ and for all $z \in \overline{O\left(S T^{-1}, S x_{0}\right)}$ ensures the existence of a common fixed point of $S$ and $T$. 
Remark 3.3. In view of Proposition 2.7, in condition (2) of Theorem 3.1 the semicompatibility of the maps can be replaced by compatibility.

The following theorem is a counterpart of Theorem 3.1 and establishes the existence of a unique common fixed point of a pair of semicompatible maps $(S, T)$ when $S$ is continuous.

Theorem 3.4. Let $S$ and $T$ be self-maps of a D-metric space $(X, D)$ satisfying conditions (1), (3) of Theorem 3.1 and

(1) the pair $(S, T)$ is semicompatible and $S$ is continuous,

(2) for all $x, y \in O\left(T^{-1} S, x_{0}\right)$ and for all $z \in X$, and for some $\phi \in \varphi$,

$$
\begin{aligned}
D(S x, S y, S z) \leq \phi \operatorname{Max}\{ & D(T x, T y, T z), D(S x, T x, T z), D(S y, T y, T z), \\
& D(S x, T y, T z), D(S y, T x, T z), D(T x, T y, S z), D(S x, T x, S z), \\
& D(S y, T y, S z), D(S x, T y, S z), D(S y, T x, S z)\} .
\end{aligned}
$$

Then $S$ and $T$ have a unique common fixed point.

Proof. For $x_{0} \in X$, construct sequences $\left\{x_{n}\right\}$ and $\left\{y_{n}\right\}$ in $X$ as in the proof of Theorem 3.1 then $S x_{n} \rightarrow u, T x_{n} \rightarrow u$. As $S$ is continuous we get $S T x_{n} \rightarrow S u$ and as $(S, T)$ is semicompatible we get $S T x_{n} \rightarrow T u$. As the limit of the sequence is unique we get

$$
S u=T u
$$

Step 4. Putting $x=x_{n}, y=x_{n}$, and $z=u$ in condition (2) we get

$$
\begin{aligned}
D\left(S x_{n}, S x_{n}, S u\right) \leq \phi \operatorname{Max} & \left\{D\left(T x_{n}, T x_{n}, T u\right), D\left(S x_{n}, T x_{n}, T u\right),\right. \\
& D\left(S x_{n}, T x_{n}, T u\right), D\left(S x_{n}, T x_{n}, T u\right), D\left(S x_{n}, T x_{n}, T u\right), \\
& D\left(T x_{n}, T x_{n}, S u\right), D\left(S x_{n}, T x_{n}, S u\right), D\left(S x_{n}, T x_{n}, S u\right), \\
& \left.D\left(S x_{n}, T x_{n}, S u\right), D\left(S x_{n}, T x_{n}, S u\right)\right\} .
\end{aligned}
$$

Taking limit as $n \rightarrow \infty$ using (3.2) and (3.11) we get

$$
D(u, u, S u) \leq \phi\{D(u, u, S u)\}<D(u, u, S u) \quad \text { if } D(u, u, S u)>0,
$$

which is a contradiction. Hence $u=S u$ and we get $u$ the common fixed point of $S$ and $T$.

The uniqueness of fixed point follows from Step 3 of Theorem 3.1.

Remark 3.5. In view of Propositions 2.7 and 2.8, condition (1) of the above theorem is not in the close reach of compatibility, weak commutativity. Thus it highlights the necessity and importance of semicompatibility in the fixed point theory of $D$-metric spaces.

In Theorem 3.1 if we take $\phi(t)=\lambda t$, for all $t \in \mathbb{R}^{+}, \lambda<1$. As $\lambda<1, \phi \in \varphi$ and we get the following. 
Corollary 3.6. Let $S$ and $T$ be self-maps of a D-metric space $(X, D)$ satisfying conditions (1), (3), (2), of Theorem 3.1 or (1) of Theorem 3.4 and

(1) for all $x, y \in O\left(T^{-1} S, x_{0}\right) \cup O\left(S T^{-1}, S x_{0}\right), z \in X$, there exists $\lambda \in[0,1)$ such that

$$
\begin{aligned}
D(S x, S y, S z) \leq \lambda \operatorname{Max}\{ & D(T x, T y, T z), D(S x, T x, T z), D(S y, T y, T z), \\
& D(S x, T y, T z), D(S y, T x, T z), D(T x, T y, S z), \\
& D(S x, T x, S z), D(S y, T y, S z), D(S x, T y, S z), \\
& D(S y, T x, S z)\} .
\end{aligned}
$$

Then $S$ and $T$ have a unique common fixed point.

In the above corollary if we take $T=I$, the identity map on $X$, then conditions (1), (2) of Theorem 3.1 are satisfied trivially and we get the following.

Corollary 3.7. Let $S$ be a self-map on a D-metric space $(X, D)$ such that for $x_{0} \in X$ the orbit $O\left(S, x_{0}\right)$ is bounded and complete and for all $x, y \in O\left(S, x_{0}\right)$, for all $z \in X$, there exists $\lambda \in[0,1)$ such that

$$
\begin{aligned}
D(S x, S y, S z) \leq \lambda \operatorname{Max}\{ & D(x, y, z), D(S x, x, z), D(S y, y, z), D(S x, y, z), \\
& D(S y, x, z), D(x, y, S z), D(S x, x, S z), D(S y, y, S z), \\
& D(S x, y, S z), D(S y, x, S z)\} .
\end{aligned}
$$

Then S has a unique fixed point.

If we restrict the contractive condition of the above corollary to the maximum of those factors which contain $z$ in the third place of function $D$, we get the following.

Corollary 3.8. Let $S$ be a self-map on a D-metric space $(X, D)$ such that for some $x_{0} \in X$ the orbit $O\left(S, x_{0}\right)$ is bounded and complete and for all $x, y \in O\left(S, x_{0}\right)$, for all $z \in X$, there exists $\lambda \in[0,1)$ such that

(A) $D(S x, S y, S z) \leq \lambda \operatorname{Max}\{D(x, y, z), D(S x, x, z), D(S y, y, z), D(x, S y, z), D(y, S x, z)\}$. Then $S$ has a unique fixed point $u$ in $X$ and $S$ is continuous at $u$.

Proof. The existence of the unique fixed point $u$ follows from Corollary 3.8. To prove the continuity, let $\left\{z_{n}\right\} \subset X$ such that $z_{n} \rightarrow u$. Putting $x=x_{m}, y=x_{m}$, and $z=z_{n}$ in condition (A) we have

$$
\begin{gathered}
D\left(S x_{m}, S x_{m}, S z_{n}\right) \leq \lambda \operatorname{Max}\left\{D\left(x_{m}, x_{m}, z_{n}\right), D\left(S x_{m}, x_{m}, z_{n}\right), D\left(S x_{m}, x_{m}, z_{n}\right),\right. \\
\left.D\left(x_{m}, S x_{m}, z_{n}\right), D\left(x_{m}, S x_{m}, z_{n}\right)\right\} .
\end{gathered}
$$

Taking limit as $m \rightarrow \infty$ we get

$$
D\left(u, u, S z_{n}\right) \leq \lambda \operatorname{Max}\left\{D\left(u, u, z_{n}\right), D\left(u, u, z_{n}\right), D\left(u, u, z_{n}\right), D\left(u, u, z_{n}\right), D\left(u, u, z_{n}\right)\right\} .
$$

Thus, $D\left(u, u, S z_{n}\right) \leq \lambda D\left(u, u, z_{n}\right)$ and hence $\lim _{n \rightarrow \infty} D\left(u, u, S z_{n}\right)=0$. Now, $D\left(S z_{n+p}\right.$, $\left.S z_{n}, u\right) \leq D\left(S z_{n+p}, u, u\right)+D\left(u, S z_{n}, u\right)$ implies that $\lim _{n \rightarrow \infty} D\left(S z_{n+p}, S z_{n}, u\right)=0$. Thus, $\left\{S z_{n}\right\}$ $D$-converges to $u$. Therefore, $S$ is continuous at $u$. 
Remark 3.9. The above corollary improves and generalizes [4, Theorem 1] in which the required domain of $x$ and $y$ in the contractive condition is needed to be the whole space $X$ while in this corollary the required domain of $x, y$ is just an orbit $O\left(S, x_{0}\right)$. Further, in the above corollary boundedness of just an orbit is taken where as in [4] the boundedness of the whole space was assumed.

Example 3.10 (of Corollary 3.8). Let $X=\left\{2^{n} \mid n \in Z\right\} \cup\{0\}=\left\{1,1 / 2,1 / 2^{2}, \ldots, 0,2\right.$, $\left.2^{2}, \ldots\right\}, d(x, y)=|x-y|$, for all $x, y \in X$. Consider

$$
D(x, y, z)=\operatorname{Max}\{|x-y|,|y-z|,|z-x|\} \quad \forall x, y, z \in X .
$$

Let $S$ be a self-map on $X$ given by $S x=x / 2$, for all $x \in X$.

Then $O(S, 1)=\left\{1,1 / 2,1 / 2^{2}, \ldots\right\}$ and $\overline{O(S, 1)}=O(S, 1) \cup\{0\}$. Thus the orbit $O(S, 1)$ is bounded and complete, however $X$ is not bounded. To show the existence of $\lambda$ of condition (A), let $M(x, y, z)=\operatorname{Max}\{D(x, y, z), D(S x, x, z), D(S y, y, z), D(x, S y, z), D(y, S x, z)\}$.

Case 1. $x, y$, and $z \in O(S, 1)$, taking $x, y$, and $z$ in descending order. We take $x=1 / 2^{n-1}$, $y=1 / 2^{n+p-1}, z=1 / 2^{n+p+t-1}$ then $S x=1 / 2^{n}, S y=1 / 2^{n+p}, S z=1 / 2^{n+p+t}$. Now,

$$
\begin{gathered}
D(S x, S y, S z)=\frac{1}{2^{n}}-\frac{1}{2^{n+p+t}}, \\
M(x, y, z)=\operatorname{Max}\left\{\frac{1}{2^{n-1}}-\frac{1}{2^{n+p+t-1}}, \frac{1}{2^{n+p-1}}-\frac{1}{2^{n+p+t-1}}, \frac{1}{2^{n}}-\frac{1}{2^{n+p+t-1}}\right\} \\
=\frac{1}{2^{n-1}}-\frac{1}{2^{n+p+t-1}}=2\left(\frac{1}{2^{n}}-\frac{1}{2^{n+p+t}}\right) .
\end{gathered}
$$

Thus, $D(S x, S y, S z)=(1 / 2) M(x, y, z)$ in this case.

Note. In case $x, z$, and $y$ are in descending order or else $z, x, y$ are in descending order, the computation can be made similarly and for $\lambda=1 / 2$ condition (A) is satisfied.

Case 2. $x, y \in O(S, 1)$ and $z=0$. We take $x=1 / 2^{n-1}, y=1 / 2^{n+p-1}, z=0$ then $S x=1 / 2^{n}$, $S y=1 / 2^{n+p}, S z=0$. Now,

$$
D(S x, S y, S z)=\frac{1}{2^{n}}, \quad M(x, y, z)=\operatorname{Max}\left\{\frac{1}{2^{n-1}}, \frac{1}{2^{n+p-1}}, \frac{1}{2^{n}}\right\}=\frac{1}{2^{n-1}} .
$$

Thus, $D(S x, S y, S z)=(1 / 2) M(x, y, z)$ in this case too.

Case 3. $x, y \in O(S, 1), z=X-O(S, 1)$, and $z \neq 0$. We take $x=1 / 2^{n-1}, y=1 / 2^{n+p-1}, z=$ $2^{m+1}$, then $S x=1 / 2^{n}, S y=1 / 2^{n+p}, S z=2^{m}$. Now,

$$
\begin{aligned}
& D(S x, S y, S z)=2^{m}-\frac{1}{2^{n+p}} \\
M(x, y, z)= & \operatorname{Max}\left\{2^{m+1}-\frac{1}{2^{n+p-1}}, 2^{m+1}-\frac{1}{2^{n}}, 2^{m+1}-\frac{1}{2^{n+p}}\right\}, \\
M(x, y, z)= & 2^{m+1}-\frac{1}{2^{n+p}}=2\left(2^{m}-\frac{1}{2^{n+p+1}}\right) \\
> & 2\left(2^{m}-\frac{1}{2^{n+p}}\right) \\
= & 2 D(S x, S y, S z) .
\end{aligned}
$$


Thus for all $x, y \in O(S, 1)$, for all $z \in X$, condition (A) is satisfied. Hence all the conditions of Corollary 3.8 are satisfied and " 0 " is the unique common fixed point of $S$.

Remark 3.11. The above example shows that the assumption of boundedness of the whole space $X$ in [4] can be removed.

In [3], Dhage et al. established the following result.

Theorem 3.12 (Dhage et al. [3]). Let $(X, D)$ be a D-metric space, $f$ a self-map on $X$. Suppose that there exists $x_{0}$ in $X$ such that $O\left(x_{0}\right)$ is bounded and $f$-orbitally complete. Suppose also that $f$ satisfies

$\left(\mathrm{A}^{\prime}\right) D(f x, f y, f z) \leq \lambda \operatorname{Max}\{D(x, y, z), D(x, f x, z)\}$, for $x, y, z \in \overline{O\left(x_{0}\right)}$ for some $0 \leq \lambda<1$. Then $f$ has a unique fixed point in $X$.

In Corollary 3.8, if we restrict the maximum to only the first two factors in the contractive condition, we get the following.

Corollary 3.13. Let $S$ be a self-map on a D-metric space $(X, D)$ such that for some $x_{0} \in X$ the orbit $O\left(S, x_{0}\right)$ is bounded and complete and for some $\lambda \in[0,1)$

$$
D(S x, S y, S z) \leq \lambda \operatorname{Max}\{D(x, y, z), D(x, S x, z)\} \quad \forall x, y \in O\left(S, x_{0}\right), z \in X
$$

Then $S$ has a unique fixed point and $S$ is continuous at it.

Remark 3.14. This corollary improves Theorem 3.1 of Dhage et al. [3] in the sense that in the contractive condition the domain of $x, y$ is just an orbit $O\left(S, x_{0}\right)$ and not its closure. Also this result corrects the above said result in the sense that the domain of $z$ in the condition must be the whole space $X$ and not just the closure of the orbit $O\left(S, x_{0}\right)$, for otherwise the uniqueness of the fixed point does not follow. The following is an example of it.

Example 3.15. Let $X=\left\{2^{n} \mid n \in Z\right\} \cup\{0\}=\left\{1,1 / 2,1 / 2^{2}, \ldots, 0,2,2^{2}, \ldots\right\}$ and $d(x, y)=$ $|x-y|$, for all $x, y \in X$. Consider

$$
D(x, y, z)=\operatorname{Max}\{|x-y|,|y-z|,|z-x|\} \quad \forall x, y, z \in X
$$

Let a self-map $S$ on the $D$-metric space $(X, D)$ be given by

$$
S x= \begin{cases}\frac{x}{2} & \text { if } x \in\left\{1, \frac{1}{2}, \frac{1}{2^{2}}, \ldots, 0\right\}, \\ \frac{2^{2}}{x} & \text { if } x \in\left\{2,2^{2}, \ldots\right\} .\end{cases}
$$

Then $O(S, 1)=\left\{1,1 / 2,1 / 2^{2}, \ldots\right\}$ and $\overline{O(S, 1)}=O(S, 1) \cup\{0\}$. Thus the orbit $O(S, 1)$ is bounded and complete. To show the existence of $\lambda$ of condition $\left(\mathrm{A}^{\prime}\right)$ see the following. 
Case 4. $x, y, z \in O(S, 1)$. We take $x=1 / 2^{n-1}, y=1 / 2^{n+p-1}, z=1 / 2^{n+p+t-1}$. As seen in Case 1 of Example 3.10, for $\lambda=1 / 2$, condition $\left(\mathrm{A}^{\prime}\right)$ is satisfied.

Case 5. $x, y \in O(S, 1)$ and $z=0$. As seen in Case 2 of Example 3.10, for $\lambda=1 / 2$, condition $\left(\mathrm{A}^{\prime}\right)$ is satisfied.

Case 6. $x=0$ and $y, z \in O(S, 1)$. We take $x=0, y=1 / 2^{n-1}, z=1 / 2^{n+p-1}$ then $S x=0$, $S y=1 / 2^{n}, S z=1 / 2^{n+p}$. Now,

$$
D(S x, S y, S z)=\frac{1}{2^{n}}, \quad \operatorname{Max}\{D(x, y, z), D(x, S x, z)\}=\operatorname{Max}\left\{\frac{1}{2^{n-1}}, \frac{1}{2^{n+p-1}}\right\}=\frac{1}{2^{n-1}} .
$$

Thus, for $\lambda=1 / 2$, condition ( $\left.\mathrm{A}^{\prime}\right)$ is satisfied.

The cases $\{x=0, y=0, z=0\}$ and $\left\{x=0, y=0, z=1 / 2^{n}\right\}$ trivially satisfy $\left(\mathrm{A}^{\prime}\right)$ for $\lambda=$ $1 / 2$. Thus for all $x, y, z \in \overline{O(S, 1)}$, condition $\left(\mathrm{A}^{\prime}\right)$ is satisfied. Hence all the conditions of [3, Theorem 3.1] are satisfied, still $S$ has two fixed points which are "0" and " 2. "

Remark 3.16. In the above example, take $x=1 / 2, y=1 / 2^{2}$, and $z=2$ then we have $S x=$ $1 / 2^{2}, S y=1 / 2^{3}$, and $S z=2$. Now,

$$
\begin{gathered}
D(S x, S y, S z)=2-\frac{1}{8}=\frac{15}{8} \\
D(x, y, z)=2-\frac{1}{2^{2}}=\frac{7}{4}, \quad D(S x, x, z)=2-\frac{1}{2^{2}}=\frac{7}{4} .
\end{gathered}
$$

Thus the contractive condition of Corollary 3.13 is not satisfied for all $x, y \in O(S, 1)$ and $z \in X$. Hence, Corollary 3.13 too cannot assure the uniqueness of a fixed point in Example 3.15.

It is to be noted that Example 3.10 is also an example of Corollary 3.13.

Corollary 3.17. Let $S$ and $T$ be self-maps of a $D$-metric space $(X, D)$ satisfying conditions (1), (2), (3) of Theorem 3.1 and

(1) let $a_{i}, i=1$ to 10 be constants such that $a_{i} \geq 0$ for all $i, \sum a_{i}<1$ and for all $x, y \in$ $O\left(T^{-1} S, x_{0}\right) \cup O\left(S T^{-1}, S x_{0}\right), z \in X$

$$
\begin{aligned}
D(S x, S y, S z) \leq & a_{1} D(T x, T y, T z)+a_{2} D(S x, T x, T z)+a_{3} D(S y, T y, T z) \\
& +a_{4} D(S x, T y, T z)+a_{5} D(S y, T x, T z)+a_{6} D(T x, T y, S z) \\
& +a_{7} D(S x, T x, S z)+a_{8} D(S y, T y, S z)+a_{9} D(S x, T y, S z) \\
& +a_{10} D(S y, T x, S z) .
\end{aligned}
$$

Then S and T have a unique common fixed point. 
Proof. Let $\sum a_{i}=\lambda(<1)$. Let

$$
\begin{aligned}
M(x, y, z)=\operatorname{Max}\{ & D(T x, T y, T z), D(S x, T x, T z), D(S y, T y, T z), \\
& D(S x, T y, T z), D(S y, T x, T z), D(T x, T y, S z), \\
& D(S x, T x, S z), D(S y, T y, S z), D(S x, T y, S z), \\
& D(S y, T x, S z)\} .
\end{aligned}
$$

Then from condition (1) of Corollary 3.17 we have

$$
\begin{aligned}
D(S x, S y, S z) & \leq \sum_{i=1}^{10} a_{i} M(x, y, z) \\
& =M(x, y, z) \sum_{i=1}^{10} a_{i} \\
& =\lambda M(x, y, z) \quad(\lambda<1) .
\end{aligned}
$$

The rest of the proof follows from Corollary 3.6.

In Corollary 3.17 if we take $T=I$, the identity map on $X$, then conditions (1), (2) of Theorem 3.1 are trivially satisfied and if we take $a_{3}=a_{4}=a_{5}=a_{6}=a_{7}=a_{9}=$ $a_{10}=0$, we get the following.

Corollary 3.18. Let $S$ be a self-map on a $D$-metric space $(X, D)$ such that for some $x_{0} \in X$, the orbit $O\left(S, x_{0}\right)$ is bounded and complete and

$$
\begin{aligned}
D(S x, S y, S z) \leq & a_{1} D(x, y, z)+a_{2} D(x, S x, z) \\
& +a_{8} D(S y, y, S z) \quad \forall x, y \in O\left(S, x_{0}\right), z \in X
\end{aligned}
$$

Then S has a unique fixed point.

Remark 3.19. The above corollary generalizes and improves [7, Theorem 1] in which the required domain of $x$ and $y$ was needed to be the whole space $X$. In this corollary the required domain of $x$ and $y$ is just an orbit $O\left(S, x_{0}\right)$ and only $z$ varies over the whole space $X$.

\section{References}

[1] B. C. Dhage, Generalised metric spaces and mappings with fixed point, Bull. Calcutta Math. Soc. 84 (1992), no. 4, 329-336.

[2] A common fixed point principle in D-metric spaces, Bull. Calcutta Math. Soc. 91 (1999), no. $6,475-480$.

[3] B. C. Dhage, A. M. Pathan, and B. E. Rhoades, A general existence principle for fixed point theorems in D-metric spaces, Int. J. Math. Math. Sci. 23 (2000), no. 7, 441-448.

[4] B. E. Rhoades, A fixed point theorem for generalized metric spaces, Int. J. Math. Math. Sci. 19 (1996), no. 3, 457-460.

[5] B. Singh and R. K. Sharma, Common fixed points via compatible maps in D-metric spaces, Rad. Mat. 11 (2002), no. 1, 145-153. 
Bijendra Singh et al. 801

[6] T. Veerapandi and K. Chandrasekhara Rao, Fixed point theorems of some multivalued mappings in a D-metric space, Bull. Calcutta Math. Soc. 87 (1995), no. 6, 549-556.

[7] _ Fixed points in Dhage metric spaces, Pure Appl. Math. Sci. 43 (1996), no. 1-2, 9-14.

Bijendra Singh: School of Studies in Mathematics, Vikram University, Ujjain 456010, Madhya Pradesh, India

E-mail address: bijendrasingh@yahoo.com

Shishir Jain: Shri Vaishnav Institute of Technology \& Science, Gram Baroli, Alwasa, Indore 453331, Madhya Pradesh, India

E-mail address: jainshishir11@rediffmail.com

Shobha Jain: MB Khalsa College, Raj Mohalla, Indore 452002, Madhya Pradesh, India

E-mail address: shobajain1@yahoo.com 


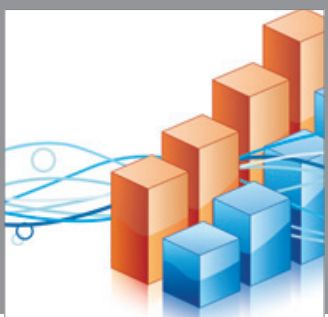

Advances in

Operations Research

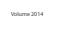

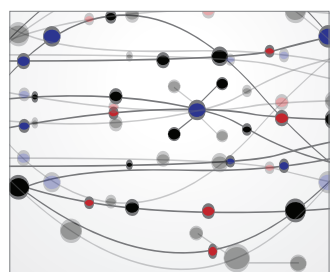

\section{The Scientific} World Journal
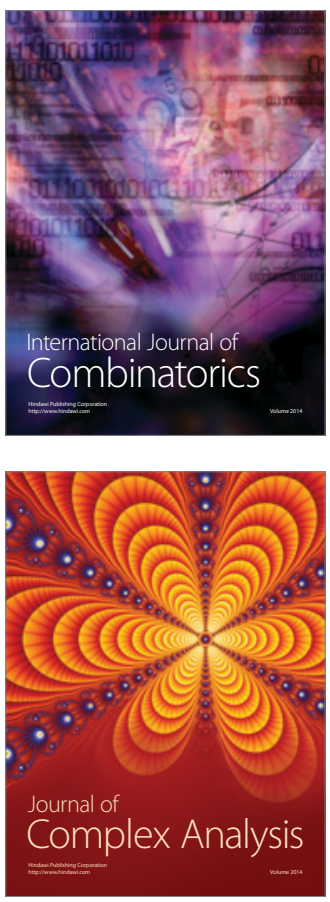

International Journal of

Mathematics and

Mathematical

Sciences
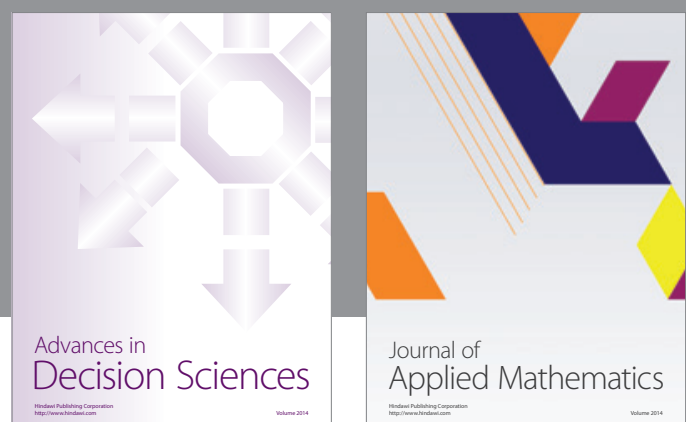

Journal of

Applied Mathematics
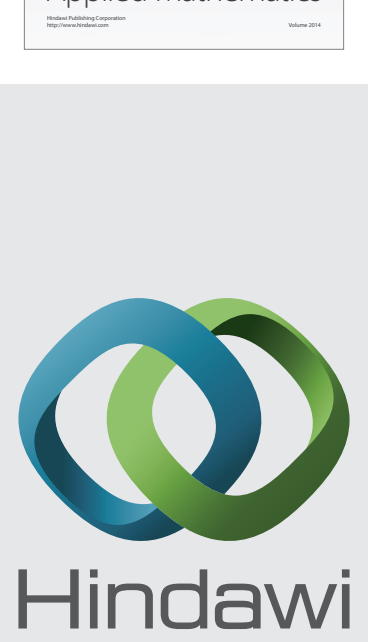

Submit your manuscripts at http://www.hindawi.com
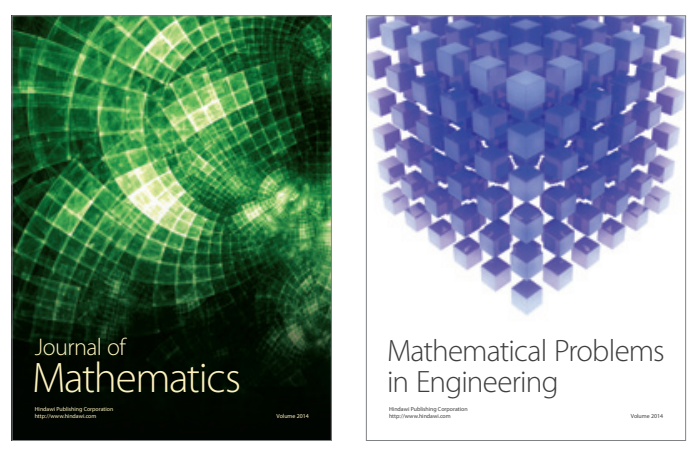

Mathematical Problems in Engineering
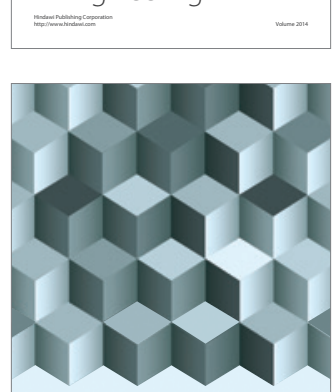

Journal of

Function Spaces
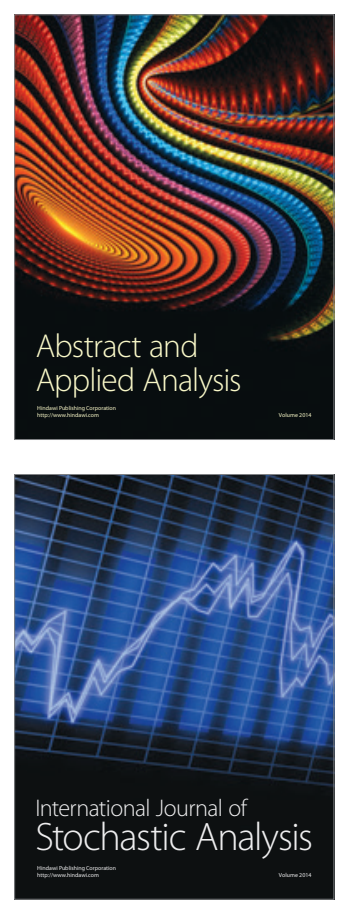

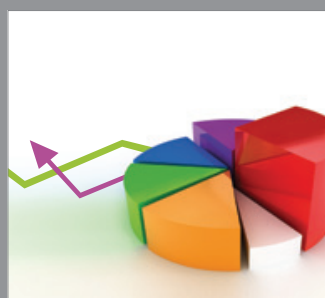

ournal of

Probability and Statistics

Promensencen
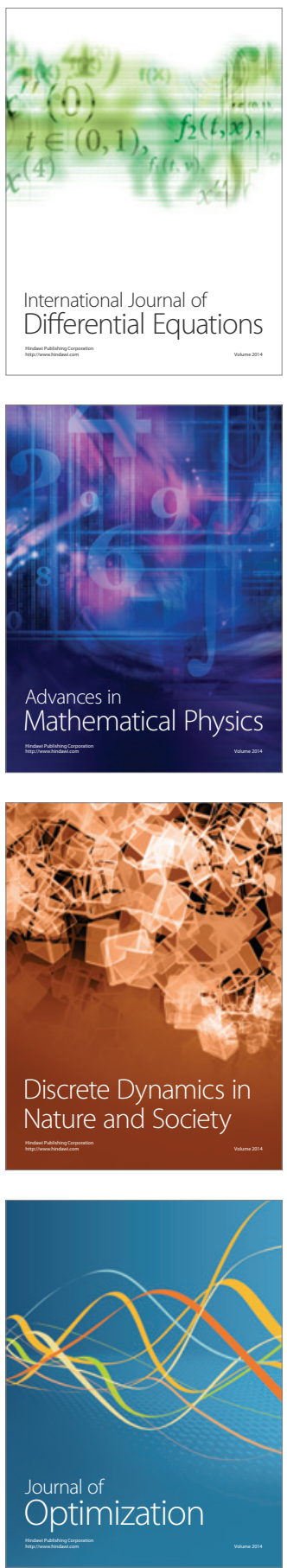